\title{
Werteffekte auf Anleiheemissionen: Eine Note für deutsche Emittenten
}
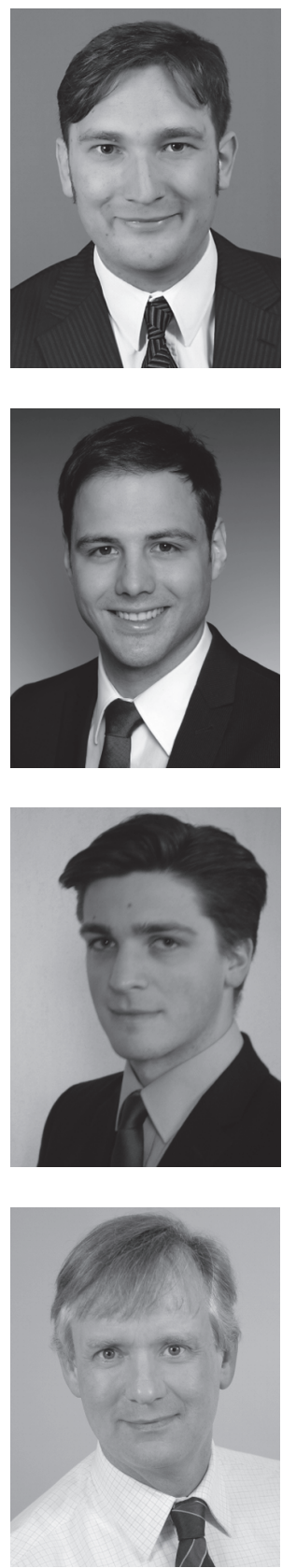

Christian Babl, Andreas Fausel, Leonard Kublman und Dirk Schiereck

Anleihenemission, Hackordnungstheorie, Trade-Off-Theorie, Ereignis-Studie, Werteffekte, Signaling

Bond issue, Pecking order theory, Trade-off theory, Event-study, Value effects, Signaling

In der finanzwirtschaftlichen Theorie wird die Wirkung von Anleiheemissionen auf den Eigenkapitalwert der Emittenten kontrovers diskutiert. Empirische Belege aus dem angelsächsische Raum legen jedoch nahe, dass es regelmässig zu negativen Renditen kommt, wodurch vor allem die Gültigkeit der Hackordnungstheorie (PeckingOrder-Theory) bestätigt wird.

In dieser Studie überprüfen wir, ob die Ergebnisse aus dem angelsächsischen Raum auch auf Deutschland übertragbar sind. Auf der Basis von 386 Anleiheemissionen im Zeitraum von 2002-2012 können wir zeigen, dass auch hier grundsätzlich negative Renditen bei Anleihebegebung zu erwarten sind. Zudem können wir durch Teilstichproben und multivariate Regression Faktoren identifizieren, die die Renditen beeinflussen. Es zeigt sich, dass die Fremdkapitalaufnahme durch Anleihen bei gesunden Unternehmen durchaus einen Mehrwert für Anleger darstellen kann, wenn der Verwendungszweck klar definiert ist.

In financial theory, the effect of bond issues on the equity value has been controversial. Empirical evidence from the Anglo-Saxon area, however, suggests that it regularly comes to negative returns, which ensures that the validity of the pecking order theory is confirmed.

In this study we examine whether the results from the Anglo-Saxon area can be transferred to Germany. On the basis of 386 bond issues in the period from 2002-2012, we can generally demonstrate that negative returns are expected from bond issue in Germany. In addition, we can construct sub-samples and a multivariate regression which helps us to identify factors that affect the returns. It turns out that borrowing through bonds can certainly add value for investors in healthy companies, if the purpose is clearly defined. 


\section{Einleitung}

Zahllose Studien beschäftigen sich in den letzten fünf Jahrzehnten mit der Suche nach einer optimalen Kapitalstruktur. Insbesondere die Frage, ob Eigen- oder Fremdkapital aufgenommen werden soll, wird kontrovers diskutiert. Um hier eine Entscheidung treffen zu können, müssen Unternehmen in der Praxis Instrumente an die Hand gegeben werden, um Nutzen und Kosten der beiden Varianten gegeneinander abwägen zu können. Dabei sind Reaktionen der Aktienkurse auf Aktien- und Anleiheemissionen als indirekte Emissionskosten nicht zu unterschätzen. Das Schwergewicht der empirischen Studien liegt im Bereich der Kapitalerhöhungen, wo auch für deutsche Emittenten regelmässig hohe negative Werteffekte für Eigenkapitalgeber bei Emissionsankündigung zu beobachten sind.

Die Auswirkungen von Fremdkapitalemissionen auf die Marktwerte des Eigenkapitals wurden dagegen gerade in Deutschland bisher nicht so ausführlich untersucht. Einschlägige Studien liegen insbesondere aus dem angelsächsischen Raum vor, wobei eine Übertragung der Evidenz von den USA und Grossbritannien mit der dort vorherrschenden starken Kapitalmarktorientierung auf das bankenorientierte Finanzsystem im deutschen Raum nicht unkritisch erscheint. Deshalb untersuchen wir nachfolgend die Aktienkursreaktionen auf Anleiheemissionen deutscher Unternehmen bzw. deutscher Muttergesellschaften für den zehn Jahre umfassenden Zeitraum von 2002 bis 2012. Wir finden für die 368 betrachteten Emissionen über das Ereignisfenster kumuliert signifikant negative abnormale Renditen von etwa $0,4 \%$.

Vom Aufbau der Arbeit her wird zunächst in Kapitel 2 ein Überblick über die theoretischen Erklärungsansätze der verschiedenen möglichen Kurseffekte aus der vorliegenden Literatur gegeben. In Kapitel 3 sollen nach einer eingehenden Beschreibung der Vorgehensweise bei der Untersuchung die Ergebnisse vorgestellt werden.

\section{Einige verbreitete theoretische Erklärungsansätze}

Die Frage, welche Werteffekte für Aktieninhaber aus einer Anleiheemission resultieren, ist eng mit der Suche nach einer vorteilhaften Kapitalstruktur verknüpft. Während sich frühe Ansätze überwiegend darauf fokussierten, minimale Kapitalkosten für das Unternehmen zu finden, um den Gesamtwert des Unternehmens zu maximieren, haben sich spätere Arbeiten auf die expliziten Effekte für Aktionäre und Fremdkapitalgeber konzentriert.

Einen Überblick zu theoretischen Ableitungen einer optimalen Kapitalstruktur geben bspw. Harris/Raviv (1991), Myers (2001) und Popescu/Visinescu (2009). Jüngere empirische Untersuchungen zur Überprüfung dieser Ansätze finden sich unter anderem bei Huang/Ritter (2009), Brounen et al. (2006) sowie Bharath et al. (2009). Die weitere knappe Strukturierung der theoretischen Erklärungsansätze folgt Eckbo (1986) und Betzer/ Limbach (2010). ${ }^{1}$

Die These, dass Anleiheemissionen keine Effekte auf den Wert des Gesamtunternehmens hervorrufen, basiert auf Modigliani/Miller (1958) zur Irrelevanz der Kapitalstruktur. Auf Basis von Arbitrageprozessen begründen sie, dass die Kapitalstruktur keinen Einfluss auf

1 Es ist dabei zu berücksichtigen, dass es sich bei den vorgestellten um eine nicht abschliessende Auswahl an Theorien handelt. Darüber hinaus existieren weitere Modelle, die in der Lage sind Werteffekte von Anleiheemissionen zu erklären. 
den Marktwert des Unternehmens hat. ${ }^{2}$ Jedoch steigen nach Modigliani/Miller die Eigenkapitalkosten mit dem Verschuldungsgrad linear, wodurch durchaus Werteffekte für Eigen- und Fremdkapitalgeber bestehen. Das Irrelevanz-Theorem behält seine Gültigkeit jedoch nur unter den sehr eingeschränkten Restriktionen eines vollkommenen Kapitalmarktes ohne Steuern und ohne Konkurskosten. Nachfolgende Arbeiten zielen darauf ab, diese Restriktionen zu lockern und realitätsnah zu formulieren. So zeigt Stiglitz (1969 und 1974), dass das Irrelevanz-Theorem auch unter der Berücksichtigung von Transaktionskosten innerhalb seiner Modelle Gültigkeit behält. Miller (1977) bezieht die steuerliche Abzugsfähigkeit von Fremdkapitalzinsen ein und lässt auch hier den Schluss zu, dass die Kapitalstruktur im Gleichgewicht keinen Einfluss auf den Unternehmenswert nimmt. Daneben existieren noch weitere Modelle, die ebenfalls unter weniger restriktive Annahmen das Irrelevanz-Theorem bestätigen.

Werden die Restriktionen zugunsten realistischerer Annahmen entschärft, indem imperfekte Märkte mit Steuern und Konkurskosten sowie asymmetrische Informationen innerhalb einer Vertragsbeziehung berücksichtigt werden, verliert das Irrelevanz-Theorem jedoch schnell an Gültigkeit. So besitzt im Rahmen der Trade-Off- und Signaling-Theorie die Kapitalstruktur einen Einfluss auf den Unternehmenswert, und Anleiheemissionen haben einen wertgenerierenden Effekt. Nach der Trade-Off-Theorie von Kraus/Litzenberger (1973) bringt ein höherer Verschuldungsgrad sowohl Vor- als auch Nachteile mit sich. Unternehmen erhöhen ihren Verschuldungsgrad so lange, bis der marginale Nutzen den marginalen Kosten der Fremdkapitalaufnahme entspricht. Dabei werden auch PrincipalAgency-Probleme erfasst, die aufgrund von asymmetrisch verteilten Informationen zwischen Fremdkapitalemittent und potentiellem Investor auftreten.

Weitere Modelle, die einen wertgenerierenden Effekt für die Anleiheemission prognostizieren, basieren auf der Signaling-Theorie. Diese leitet sich überwiegend aus den Arbeiten von Akerlof (1970) und Arrow (1972) zum Produkt- und Arbeitsmarkt ab und wurde durch Spence (1973) in ein Gleichgewichtsmodell übertragen. Hier liegt auch die Annahme einer asymmetrischen Informationsverteilung zwischen den Vertragspartnern zugrunde. Investoren versuchen aus Entscheidungen des Managements Informationen über die Situation des Unternehmens zu gewinnen. Diesen Zusammenhang kann wiederum das Management nutzen, um den Investoren glaubhafte Informationen über die Qualität der Investitionen zu übermitteln. Als Signal dient die Zusammensetzung der gewählten Kapitalstruktur. Ross (1977) zeigt die Möglichkeit auf, dass Manager als positives Signal für die aktuelle Ertragslage und Zukunftsaussichten einen höheren Verschuldungsgrad wählen. Zusammenfassend lässt sich sagen, dass nach der Signaling-Theorie eine Fremdkapitalaufnahme ein positives Indiz für mögliche Eigenkapitalgeber ist, und damit eine positive Reaktion der Aktienmärkte begründbar wäre.

Im Gegensatz zu den bisher genannten Ansätzen versuchen Unternehmen nach der Pecking-Order-Theorie von Myers/Majluf (1984) nicht, eine fest definierte optimale Kapitalstruktur zu erreichen. Anstelle dessen wird eine Rangordnung innerhalb der Finanzierungsmöglichkeiten eines Unternehmens angenommen, wobei von Fall zu Fall entschieden wird, welche Finanzierungsmöglichkeit gewählt wird. Eine externe Kapitalaufnahme stellt ein negatives Signal für Aktionäre dar, da sie entweder mit einer Kapitalverwässerung oder

2 Alternativ kann die Irrelevanz des Verschuldungsgrades auch mit Hilfe der Wertadditivität begründet werden (vgl. hierzu Franke/Hax (2004)). 
mit zusätzlichen Zinslasten verbunden ist. Empirische Untersuchungen von Rajan/Zingales (1995), Fama/French (2002) und Drobetz/Fix (2005) können damit übereinstimmend eine negative Abhängigkeit des gewählten Fremdfinanzierungsgrades von der Profitabilität eines Unternehmens nachweisen. Ist keine Innenfinanzierung möglich, steht das Unternehmen vor der Wahl, entweder Eigenkapital oder Fremdkapital aufzunehmen. Der PeckingOrder-Theorie zufolge zieht das Unternehmen hier neues Fremdkapital der Emission von Eigenkapital vor. Bezüglich der Werteffekte des Eigenkapitals ist nach Eckbo (1986) und Shyam-Sunder (1991) bei Aktienemissionen mit deutlich negativeren Kurskorrekturen als bei Anleihen zu rechnen. Diese sind bei Fremdkapitalemissionen nur relativ gering bzw. nicht nachweisbar.

Neben der generellen Aussage über die Wirkung einer Fremdkapitalaufnahme auf den Marktwert des Eigenkapitals befassen sich zahllose Untersuchungen mit einzelnen Faktoren, die das Ausmass einer Kursreaktion erklären sollen und nachfolgend ebenfalls in die empirische Analyse einbezogen werden:

Zinssatz: Taggart (1977) und Marsh (1982) sowie Brounen et al. (2006) stellen fest, dass das aktuelle Zinsniveau einen grossen Einfluss auf die Entscheidung hat, ob ein Unternehmen Eigenkapital oder Fremdkapital emittiert. Auch Krishnaswami/Yaman (2007) untersuchen diesen Sachverhalt empirisch und gehen davon aus, dass bei einer Emission von Fremdkapital in einer Phase hohen Zinsniveaus mit ebenfalls höheren Zinskosten zu rechnen ist. Damit steigt die Gefahr eines Financial Distress, und wegen des höheren Financial-Distress-Risikos ist in Phasen hoher Zinssätze mit negativeren Kursreaktionen zu rechnen.

Konjunktur: Sowohl Moore (1980) als auch Choe et al. (1993) stellen fest, dass in Phasen schwächerer Konjunktur weniger Eigenkapital emittiert wird. Im Gegensatz dazu bleibt die Anzahl der Fremdkapitalemissionen gleich, was sich mit dem von der Konjunktur relativ unabhängigen (Re-) Finanzierungsbedarf der Unternehmen begründen lässt. Trotzdem sind bei Emissionen in Phasen schwacher Konjunktur negativere abnormale Renditen zu erwarten als bei Aufschwung, da hier das Financial Distress-Risiko höher ist.

Rating: Bei Anleiheemissionen von Unternehmen mit einem Rating, das schlechter als $\mathrm{BB}+(\mathrm{S} \& \mathrm{P})$, bzw. Ba1 (Moody's) ist, wird von High-Yield-Bonds gesprochen. Da solche Unternehmen für die Investoren mit einem relativ grossen Risiko behaftet sind, müssen die Anleihen zu einem höheren Zins begeben werden. Daraus resultieren für den Emittenten erhöhte Kapitalkosten.

Währung: Die Aufnahme von Fremdkapital in einer Fremdwährung ist ein wichtiges Instrument zur Absicherung (Hedging) gegenüber Währungsrisiken auf der Vermögensund Einnahmeseite. Smith/Stulz (1985) zeigen, dass der Unternehmenswert durch Hedging gesteigert werden kann, weil dadurch Steuerzahlungen reduziert werden können. Die ebenfalls dort aufgestellte These, dass Hedging Financial Distress-Kosten verringert, ist umstritten. ${ }^{3}$ Ein Unternehmen, das eine Anleiheemission aus Gründen geringerer Platzierungskosten in Erwägung zieht, befindet sich jedoch in einem Trade-Off zwischen günstigeren Fremdkapitalkosten und einen durch Wechselkursrisiken verursachten Anstieg der Finanzierungsrisiken. Aufgrund der Möglichkeiten von Hedging zur Fremdkapitalkosten-

3 Howton/Perfect (1998), Graham/Rogers (2002) finden nur einen geringen oder keinen Effekt von Hedging auf Financial-Distress-Kosten, während Berkman/Bradbury (1996) einen Zusammenhang feststellen. 
reduktion können in Fremdwährung denominierte Anleihen wertgenerierender als Anleihen in der lokalen Währung sein.

Emissionsvolumen: Wegen der herrschenden Informationsasymmetrien sind die Aktionäre eines Unternehmens meistens gezwungen, Investitions- und Finanzierungsentscheidungen $\mathrm{zu}$ interpretieren. Nach der Pecking-Order-Theorie impliziert eine Fremdkapitalaufnahme eines Unternehmens, dass die momentane Ertragslage eine Innenfinanzierung des aktuellen Kapitalbedarfes nicht zulässt. Infolge dieses negativen Signals werden die Aktienkurse an den nun geringer geschätzten Marktwert des Unternehmens angepasst und damit nach unten korrigiert. ${ }^{4}$ Dabei lässt sich an dem jeweiligen Volumen der Emission der Umfang der benötigten Aussenfinanzierung ablesen.

Freefloat: Eine Anteilseigner-Struktur, in der sich grosse Teile der Aktien im Besitz von Grossaktionären befinden, birgt Vorteile für den Wert der Aktie. Insbesondere hängt dies damit zusammen, dass Grossaktionäre mehr Kontrolle und Macht auf das Unternehmen ausüben können - beispielsweise in Form von Sitzen im Aufsichtsrat. Wie im Zusammenhang mit der Trade-Off-Theorie beschrieben, sind gerade in grösseren Unternehmen Principal-Agency- Probleme und damit verbundene Kosten zu beobachten. Nach der eben dargelegten Argumentation verhält sich die Kontrolle der Manager durch die Anteilseigner invers zum Freefloat eines Unternehmens. Es lässt sich also davon ausgehen, dass bei geringerem Freefloat weniger Principal-Agency-Probleme auftreten.

Emittentengrösse: Warner (1977) stellt bereits fest, dass grössere Unternehmen mit einem geringeren Financial-Distress-Risiko in Verbindung gebracht werden, da sie in der Regel stärker diversifiziert sind. Daraus lässt sich ein positiver Zusammenhang zwischen Unternehmensgrösse und Fremdkapitalanteil ableiten (Psillaki/Daskalakis, 2009). Auf unsere Problematik übertragen bedeutet dies, dass der Markt bei grösseren Unternehmen positiver auf neue Fremdkapitalemissionen reagiert als bei kleineren. Rajan/Zingales (1995), sowie Graham/Harvey (2001) folgend kann man die Unternehmensgrösse auch als indirektes Mass für Informationsasymmetrien zwischen Unternehmen und Investoren sehen. Bei grossen Unternehmen sind die Informationsasymmetrien deutlich geringer, da sich beispielsweise viele Analysten regelmässig mit den Unternehmen beschäftigen. Die Qualität und Quantität der Informationen, die externe Investoren über ein Unternehmen haben, korreliert also positiv mit der Unternehmensgrösse.

Verwendungszweck: Man kann davon ausgehen, dass desto geringere Informationsasymmetrien vorliegen, je genauer der Verwendungszweck des Fremdkapitals bekannt ist. Ausserdem sollte es einen positiveren Einfluss auf die Aktienkurse haben, wenn das Unternehmen neues Fremdkapital für neue Projekte benötigt, anstatt es zur Refinanzierung alter Schulden zu verwenden. Diese Argumentation setzt voraus, dass neue Projekte einen positiven Kapitalwert besitzen und zu einer Steigerung des Unternehmenswerts führen. Gegensätzlich zu dieser Argumentation erwarten Myers/Majluf (1984) sowie Miller/Rock (1985), dass der Finanzierung neuer Projekte dienende Emissionen einen negativeren Einfluss auf Aktienkurse haben, während Ross (1977) von dem Gegenteil ausgeht.

4 Vgl. dazu Myers/Majluf (1984), sowie Miller/Rock (1985). 


\section{Untersuchungsmethodik und Datensatz}

\subsection{Untersuchungsdesign / Untersuchungsmethodik}

Im Rahmen dieser Untersuchung werden Aktienkursreaktionen auf die Ankündigung von Anleiheemissionen analysiert. Als empirische Untersuchungsmethode wird dazu eine Ereignisstudie (Event Study) nach Fama et al. (1969) gewählt. Die Event Study untersucht Werteffekte, die den Anteilseignern durch das Eintreten eines vorab zu definierenden Ereignisses entstehen. Bei dem Ereignis handelt es sich in diesem Fall um die Anleiheemission, die Werteffekte werden als abnormale Renditen (Abnormal Return) bezeichnet. Eine ausführliche Darstellung der Methodik findet sich bspw. bei MacKinlay (1997).

Da Störereignisse (Confounding Events) sich in einem Modell kaum operational berücksichtigen lassen, bleiben nahfolgend die Anleiheemissionen unberücksichtigt, bei denen unternehmens- oder branchenrelevante Informationen innerhalb des Untersuchungszeitraums der Emission bekannt werden.

In Anlehnung an vergleichbare Studien wurde eine Ereignisperiode gewählt, die 10 Handelstage vor dem Ereignistag beginnt und 10 Handelstage nach dem Ereignis endet. Damit können sowohl Effekte durch Insiderhandel und Gerüchte, die vor dem Ereignistag auftreten, als auch eventuelle spätere Gegenreaktionen abgebildet werden. Somit umfasst die Gesamtdauer des Event-Fensters 21 Handelstage. Neben dem Ereignisfenster muss auch die Schätzperiode festgelegt werden, für die 200 Handelstage vor der Ereignisperiode gewählt wurde.

Um die Ergebnisse der Event Study zu evaluieren, werden statistische Testverfahren herangezogen. Dabei werden sowohl die Anforderungen an die linearen Regressionen als auch die Signifikanz der abnormalen Renditen geprüft. Zur Überprüfung, ob eine Autorkorrelation bei den jeweiligen Ereignissen vorliegt, wurde der Durbin-Watson-Test verwendet.

Zusätzlich kommt ein T-Test nach Boebmer et al. (1991) zur Anwendung, um mögliche Änderungen der Varianz bei Event-Eintritt zu berücksichtigen. Werden die abnormalen Renditen zweier Teilstichproben verglichen, dann geschieht dies auf Basis der Varianzanalyse bzw. des T-Tests.

Mit einem multiplen Regressionsmodell wird der Erklärungsgehalt der einzelnen Variablen zu den abnormalen Renditen analysiert. Die Regressionskoeffizienten der j Variablen und die Konstante werden mittels der OLS Methode geschätzt. Als erklärende Variable wird die standardisierte kumulierte abnormale Rendite (SCAR) verwendet, um dadurch einer möglichen Heteroskedastizität vorzubeugen. Auf Multikollinearität wird durch den Variance Inflation Factor geprüft. Das Signifikanzniveau der Regressionskoeffizienten wird im Rahmen des T-Tests ermittelt.

\subsection{Datensatz und deskriptive Statistik}

Ein Ereignis im Sinne unserer Untersuchung erfüllt folgende Kriterien: ${ }^{5}$

5 Die Höhe des Emissionsvolumens von mindestens 5 Prozent der Marktkapitalisierung dient dazu, dass die Emission eine Mindestgrösse besitzen muss, um als kursrelevant eingestuft werden zu können. Das Mindestemissionsvolumen von 200 Mio. Euro wurde als Kriterium eingeführt, um eine möglichst grosse Heterogenität von Emittenten in der Stichprobe zu erhalten. 
- Es handelt sich bei dem Ereignis um eine Ankündigung zur Begebung einer Anleihe im Zeitraum zwischen dem 1.1.2002 und dem 24.10.2012.

- Die Muttergesellschaft des Emittenten hat ihren Hauptsitz in Deutschland.

- Die Muttergesellschaft oder der Emittent ist an einer deutschen Börse notiert, sodass Aktienkurse über einen Zeitraum von 210 Handelstage vor bis 10 Handelstage nach der Emission verfügbar sind und ein handelbarer Streubesitz existiert.

- Das Emissionsvolumen entspricht mindestens 5 Prozent der Marktkapitalisierung der Muttergesellschaft.

- Das Emissionsvolumen beträgt mindestens 200 Mio. Euro.

\begin{tabular}{lccccc}
\hline Jahr & Anzahl & $\begin{array}{c}\text { Volumen } \\
\text { in [Mio. } € \text { ] }\end{array}$ & $\begin{array}{c}\text { Vol. Mittel } \\
\text { in [Mio. } €]\end{array}$ & $\begin{array}{c}\text { Vol. } \\
\text { in [\%] }\end{array}$ & $\begin{array}{c}\text { Anzahl } \\
\text { in [\%] }\end{array}$ \\
\hline 2002 & 18 & 24,298 & 1,350 & 9 & 5 \\
2003 & 41 & 45,632 & 1,113 & 8 & 11 \\
2004 & 61 & 53,308 & 874 & 6 & 17 \\
2005 & 61 & 71,011 & 1,164 & 8 & 17 \\
2006 & 65 & 83,004 & 1,277 & 9 & 18 \\
2007 & 35 & 41,882 & 1,197 & 8 & 3 \\
2008 & 10 & 18,382 & 1.838 & 13 & 4 \\
2009 & 14 & 26,282 & 1,877 & 13 & 7 \\
2010 & 27 & 33,190 & 1,229 & 8 & 5 \\
2011 & 19 & 20,126 & 1,059 & 7 & 5 \\
2012 & 17 & 28,537 & 1,679 & 11 & 100 \\
\hline Gesamt & 368 & 445,652 & 1,332 & 100 & \\
\hline
\end{tabular}

Tabelle 1: Deskriptive Statistik: betrachtete Anleiheemissionen pro Jahr

Werden Anleihen in mehrere Tranchen emittiert, dann kann nur der Gesamteffekt gemessen werden. In diesen Fällen werden die einzelnen Tranchen zu einer Emission zusammengefasst und entsprechend berücksichtigt. Zudem werden hier nur reine Anleihen betrachtet, Wandelanleihen bleiben aussen vor. Aus 10.773 Anleiheemissionen zwischen 1.1.2002 und 24.10.2012 wird nach den oben genannten Kriterien aussortiert, sodass 368 relevante Emissionen übrig bleiben.

Durch die Selektion möglichst grosser und möglichst kursrelevanter Emissionen entsteht ein gewisser Bias zugunsten von Emissionen der Finanzbranche, die die aufgestellten Kriterien eher erfüllen. Es ist aber darauf zu achten, dass die Motivation der Finanzbranche vor allem bei der Refinanzierung liegt. Im Rahmen der multivariaten Analyse wird deshalb gesondert auf die Übertragung der Ergebnisse für die Finanzbranche einzugehen sein. Zudem muss darauf geachtet werden, dass die Ergebnisse nicht unbedingt auf kleinere Anleihenbegebungen übertragen werden können.

Informationen zu einzelnen Emissionen werden der Datenbank Thomson ONE Banker entnommen. Zeitreihen wie Aktienkurse, Marktkapitalisierung oder das Bruttoinlandprodukt werden mit Hilfe von Datastream generiert. Um Confounding Events aufzuspüren, wird eine Websuche durchgeführt. Auf Basis von Google News wird dabei jeweils ein Zeitraum von 2 Wochen vor und 2 Wochen nach der jeweiligen Emission für das entsprechende Unternehmen abgedeckt. Durch Variationen von Marktportfolio, Länge der Schätzperiode und des Ereignisfensters, Höhe des absoluten Emissionsvolumens und Höhe 
des Emissionsvolumens in Relation zur Marktkapitalisierung wird die Robustheit der Ergebnisse überprüft. Da sich die Ergebnisse gegenüber der Variation der Parameter stabil verhalten, können die Resultate verallgemeinert werden. Als Marktportfolio wird der STOXX Europe 600 gewählt.

\section{Ergebnisse der Event Study}

Die Ergebnisse der Ereignisstudie auf Basis des STOXX Europe 600 sind in Tabelle 2 abgebildet. Die CAAR sind in der zweiten Spalte jeweils für verschiedene Ereignisfenster zusammengefasst. Zudem werden die zugehörigen Teststatistiken nach Boebmer et al. (1991) in der dritten Spalte dargestellt. Es ist zu erkennen, dass die CAAR für alle Ereignisfenster negativ sind und zumindest kurzfristig auch signifikante Niveaus erreichen.

\begin{tabular}{ccc}
\hline Ereignisfenster & CAAR $[\%]$ & T-Test nach BMP \\
\hline$[-10 ; 0]$ & $-0,518 * *$ & $-2,412$ \\
{$[-5 ; 0]$} & $-0,344 *$ & $-1,757$ \\
{$[-3 ; 0]$} & $-0,406 * *$ & $-2,368$ \\
{$[-2 ; 0]$} & $-0,266$ & $-1,351$ \\
{$[-1 ; 0]$} & $-0,179$ & $-1,047$ \\
{$[0]$} & $-0,163 * *$ & $-2,170$ \\
{$[-10 ; 10]$} & $-0,634$ & $-1,539$ \\
{$[-5 ; 5]$} & $-0,415$ & $-1,347$ \\
{$[-3 ; 3]$} & $-0,509 * *$ & $-2,418$ \\
{$[-2 ; 2]$} & $-0,407 *$ & $-1,963$ \\
{$[-1 ; 1]$} & $-0,226$ & $-1,288$ \\
{$[0 ; 10]$} & $-0,279$ & $-0,512$ \\
{$[0 ; 3]$} & $-0,304 *$ & $-1,965$ \\
{$[0 ; 1]$} & $-0,211 * *$ & $-2,106$ \\
{$[-10 ; 1]$} & $-0,566 * *$ & $-2,501$ \\
{$[2 ; 10]$} & $-0,068$ & 0,280 \\
\hline
\end{tabular}

$* * *$ signifikant auf dem 1\%-Niveau, ** signifikant auf dem 5\%-Niveau, * signifikant auf dem 10\%-Niveau

Tabelle 2: CAAR der durchgeführten Event Study

Der zeitliche Verlauf der CAAR ist in Abbildung 1 dargestellt. Dabei fällt auf, dass der grösste Anteil des Werteffekts bereits vor der Bekanntgabe der Emission bzw. am Ankündigungstag selbst realisiert wird. Erwartungsgemäss fällt die CAAR auch am Folgetag der Ankündigung negativ aus, sodass im Ereignisfenster [-10; 1] die CAAR -0,566 Prozent beträgt. Die abnormalen Renditen dieser Periode sind ebenfalls hochsignifikant, wohingegen die Wertentwicklung im nachfolgenden Zeitraum [2; 10] nicht signifikant von Null abweicht. Zudem sind bereits vor dem Ankündigungstag Wertentwicklungen zu beobachten. Diese könnten auf Marktteilnehmer zurückzuführen sein, die über nicht öffentliche Infor- 
mationen verfügen und die Wertentwicklung antizipieren. Die Beobachtung unterstützt die Annahme starker Informationseffizienz, d.h. der Markt preist auch Insiderinformation effizient ein.

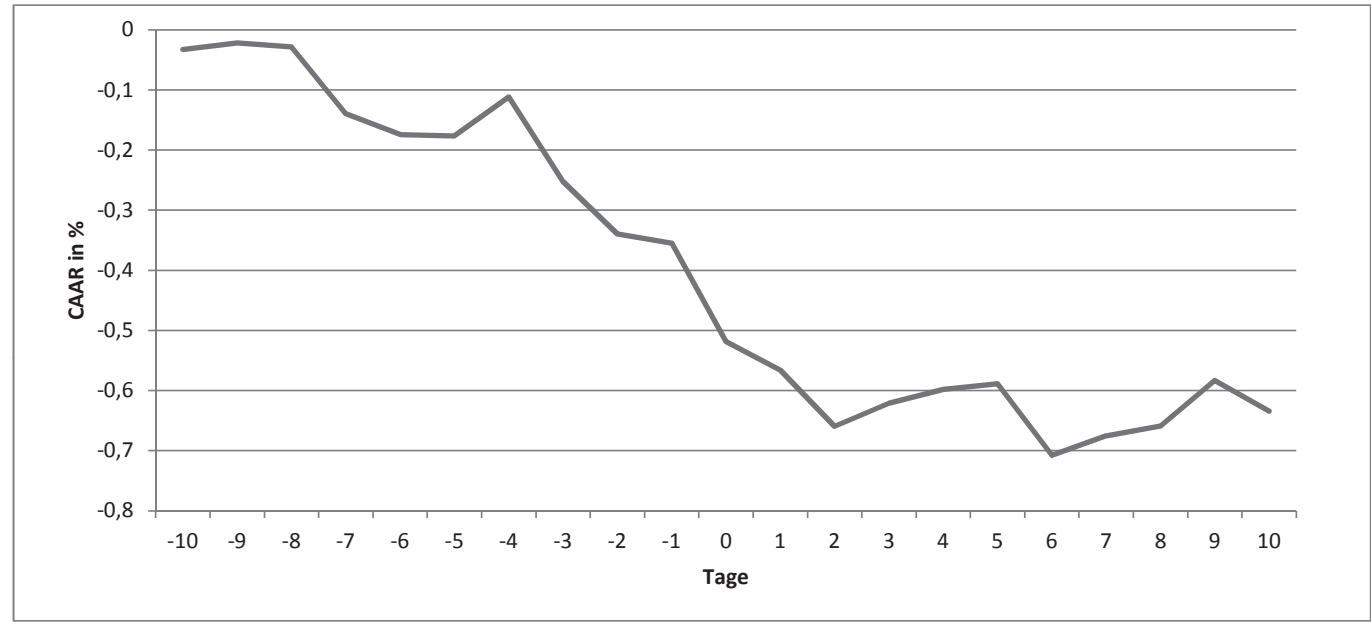

Abbildung 1: CAAR im Zeitverlauf

Die negativen CAAR am Ankündigungstag in Höhe von -0,163 und für den Zeitraum [0; -1] in Höhe von 0,211 decken sich vor allem mit den jüngeren Untersuchungen von Krishnaswami/Yaman (2007) sowie Dann/Mikkelson (1984). In Tabelle 3 befindet sich eine Übersicht von Studien und deren Ergebnisse, in welchen ähnliche Untersuchungen für den US-amerikanischen Markt durchgeführt wurden. Auch in einem traditionell eher bankorientierten Finanzsystem wie dem deutschen Kapitalmarkt wird die Emission von Anleihen nicht generell kritischer gewertet als in marktorientierten angelsächsischen Finanzsystemen. Die Ergebnisse lassen sich durch die Pecking-Order-Theorie oder durch ein Entfernen von der optimalen Kapitalstruktur im Sinne der Trade-Off-Theorie erklären.

\begin{tabular}{lcccc}
\hline \multicolumn{1}{c}{ Autor } & $\begin{array}{c}\text { Untersuchungs- } \\
\text { zeitraum }\end{array}$ & $\begin{array}{c}\text { Stichpro- } \\
\text { bengrösse }\end{array}$ & Ereignisfenster & CAAR \\
\hline Krishnaswami/Yaman (2007) & $1983-1998$ & 1.862 & {$[-1 ; 0]$} & $-0,151 * *$ \\
& & & {$[0]$} & $-0,122 * *$ \\
& & {$[0 ; 1]$} & $-0,197 * *$ \\
Dann/Mikkelson (1984) & $1970-1979$ & 150 & {$[-1 ; 1]$} & $-0,225 * *$ \\
Mikkelson/Partch (1986) & $1972-1982$ & 360 & {$[-1 ; 0]$} & $-0,37 *$ \\
Shyam-Sunder (1991) & $1980-1984$ & 297 & {$[-1 ; 0]$} & $-0,23$ \\
\hline
\end{tabular}

*** signifikant auf dem 1\%-Niveau, ** signifikant auf dem 5\%-Niveau, * signifikant auf dem 10\%-Niveau

Tabelle 3: Studien zur Anleihemission 
Tabelle 4 zeigt die Ergebnisse der Analyse einzelner Einflussgrössen wie Rating, Währung, Makro-Branche (Banken vs. Rest) und Verwendungszweck. Dazu wird die gesamte Stichprobe jeweils in zwei Gruppen aufgeteilt und mittels Zweistichproben-T-Test auf Gleichheit überprüft. Es werden aus einer grösseren Auswahl an Ereignisfenstern nur die signifikanten Ergebnisse dargestellt.

\begin{tabular}{|c|c|c|c|c|c|}
\hline \multirow{2}{*}{$\begin{array}{l}\begin{array}{c}\text { Untersuchungs-ge- } \\
\text { genstand }\end{array} \\
\text { Rating }\end{array}$} & \multirow[t]{2}{*}{$\begin{array}{l}\text { Ereignis- } \\
\text { fenster }\end{array}$} & \multicolumn{2}{|c|}{$\begin{array}{c}\text { CAAR } \\
\text { (Stichprobengrösse) }\end{array}$} & \multirow[t]{2}{*}{$\begin{array}{c}\text { CAAR } \\
\text { Differenz }\end{array}$} & \multirow[t]{2}{*}{ p-Wert } \\
\hline & & $\begin{array}{l}\text { High-Yield } \\
(20)\end{array}$ & $\begin{array}{c}\text { Investment-Grade } \\
(348)\end{array}$ & & \\
\hline & {$[-3 ; 0]$} & $-1,097$ & $-0,367$ & $-0,730 *$ & 0,0997 \\
\hline & {$[-5 ; 0]$} & $-1,344$ & $-0,286$ & $-1,057 * *$ & 0,0381 \\
\hline \multirow[t]{4}{*}{ Währung } & & $\begin{array}{l}\text { Euro } \\
(292)\end{array}$ & $\begin{array}{l}\text { Fremdwährung } \\
(76)\end{array}$ & & \\
\hline & {$[-1 ; 1]$} & $-0,334$ & 0,043 & $-0,377^{*}$ & 0,0819 \\
\hline & {$[-1 ; 0]$} & $-0,269$ & 0,040 & $-0,309 *$ & 0,0635 \\
\hline & {$[-2 ; 0]$} & $-0,364$ & 0,023 & $-0,387^{*}$ & 0,0867 \\
\hline \multirow[t]{3}{*}{ Makro-Branche } & & $\begin{array}{c}\text { Finanzwirtschaft } \\
(272)\end{array}$ & $\begin{array}{c}\text { andere Branchen } \\
(96)\end{array}$ & & \\
\hline & {$[-10 ; 10]$} & $-0,923$ & 0,186 & $-1,110 *$ & 0,0598 \\
\hline & {$[0 ; 10]$} & $-0,466$ & 0,253 & $-0,719 *$ & 0,0824 \\
\hline \multirow[t]{2}{*}{ Verwendungszweck } & & $\begin{array}{l}\text { anderer Verwen- } \\
\text { dungszweck (29) }\end{array}$ & $\begin{array}{c}\text { Refinanzierung } \\
(339)\end{array}$ & & \\
\hline & {$[0 ; 1]$} & 0,291 & $-0,253$ & $0,544 *$ & 0,0828 \\
\hline
\end{tabular}

Tabelle 4: signifikante CAAR für ausgewählte Teilstichproben

Bei der Einflussgrösse Rating werden 20 Emissionen aus dem High-Yield-Bereich 348 Emissionen mit Investment-Grade gegenübergestellt. Die Stichprobe ist zwar mit 20 Emissionen auf der High-Yield-Seite sehr klein, allerdings sind die Ergebnisse auch sehr eindeutig. $\mathrm{Zu}$ beachten ist im Ereignisfenster [-5; 0] eine auf 5-Prozent-Niveau signifikante Differenz zwischen den beobachteten CAAR von -1,057 Prozent. Im Vergleich dazu stellt Shyam-Sunder (1991) keinen signifikanten Zusammenhang zwischen der Höhe des Ratings und den abnormalen Renditen fest. Unterschiede zwischen 292 Emissionen in Euro bzw. 76 in Fremdwährungen zeigen sich deutlich im Fenster [-2; 0]. Hier findet man eine Differenz zwischen den abnormalen Renditen bei Euro und Fremdwährungen von -0,387 Prozent. Emissionen in Fremdwährungen bringen sogar durchgängig leicht positive abnormale Renditen. Beim Vergleich von Finanzwirtschaft (272 Emissionen) mit anderen Branchen (96 Emissionen) finden sich zwar auch eine schwach-signifikante Differenz von -1,11 Prozent zwischen den abnormalen Renditen der beiden Gruppen, allerdings tritt diese im Fenster [-10; 10] auf. Insgesamt sind Signifikanzen bei dieser Teilstichprobe nur in den grössten betrachteten Ereignisfenstern zu beobachten. 
Mit 339 Emissionen dient ein grosser Teil der betrachteten Fremdkapitalemissionen nicht genauer definierten Zwecken oder der Refinanzierung, während bei lediglich 29 Emissionen ein genauerer Verwendungszweck bzw. die Investition in neue Projekte angegeben ist. Bei einer Differenz von 0,544 Prozentpunkte zwischen den abnormalen Renditen beider Gruppen im Fenster [0;1] sind deutlich positive Renditen für Emissionen mit einem Verwendungszweck, der keine Refinanzierung beinhaltet, beobachtbar. Im Vergleich dazu können Krishnaswami/Yaman (2007) in einer multivariaten Untersuchung für den Verwendungszweck keinen signifikanten Einfluss nachweisen.

Zur weiteren Erklärung der beobachteten Werteffekte werden multivariate Regressionsmodelle eingesetzt. Es werden die Variablen Währung, Makro-Branche, Rating, Verwendungszweck, Marktkapitalisierung, Emissionsvolumen, Freefloat, Konjunktur, Zinssatz, Wachstumsmöglichkeiten, Financial-Distress-Kosten und Agency-Kosten berücksichtigt. Die ersten vier Variablen werden als Dummy Variablen modelliert. Den Ausprägungen Euro, Finanzsektor, High Yield und ein Verwendungszweck, der nicht zur Refinanzierung dient, werden jeweils die Eins zugeordnet. Zudem wird der Literatur folgend unterstellt, dass der Effekt der Marktkapitalisierung (MK) mit steigendem Wert abnimmt. Deshalb wird die Marktkapitalisierung logarithmiert. Financial-Distress-Kosten werden durch den Zinsdeckungsgrad modelliert. Die Masszahl für die Konjunktur ist das reale Wirtschaftswachstum gemessen am Bruttoinlandsprodukt. Als risikoloser Zinssatz wird die Effektivverzinsung von deutschen Staatsanleihen mit einer Laufzeit von fünf Jahren gewählt. Die Laufzeit entspricht zugleich dem Median der angekündigten Emissionen. Wachstumsmöglichkeiten werden vom Market-to-Book-Verhältnis ausgedrückt. Die Relation EBIT zu Zinsaufwendungen drückt die Konkurswahrscheinlichkeit aus. Agency Kosten werden mit Hilfe des Excess-Cash-Flows gemessen, das ist der Geldstrom, der den Managern zur freien Verfügung steht.

Aus Tabelle 5 sind die Ergebnisse zu entnehmen. Bei den Einflussgrössen Währung und Makro-Branche können die Ergebnisse aus dem vorherigen Abschnitt nicht bestätigt werden. Die Bekanntmachung eines Vorhabens, das nicht der Refinanzierung dient, ruft aber wieder einen positiven Werteffekt hervor. Eine grosse Marktkapitalisierung hat eine signifikante negative Wirkung. Dies könnte darauf zurückzuführen sein, dass kleine Unternehmen einen schwierigeren Zugang zum Kapitalmarkt haben als vergleichsweise grosse Unternehmen. Eine andere Erklärung könnte darauf zurückzuführen sein, dass mit zunehmender Marktkapitalisierung auch zunehmend Unternehmen der Finanzbranche abgebildet werden, welche wie bereits gezeigt, negativere Renditen ausweisen. Schafft es ein kleines Unternehmen doch, eine Anleihe am Markt zu platzieren, dann kann das von den Investoren als Erfolg bzw. als weniger negativ wahrgenommen werden. Bei Gültigkeit der Pecking-Order-Theorie ist dies vor allem dann der Fall, wenn das Unternehmen sonst auf noch teurere Finanzierungsmöglichkeiten zurückgreifen müsste.

Das Emissionsvolumen hat wider Erwarten einen positiven signifikanten Werteffekt. Hierfür könnten mögliche Skaleneffekte, die aufgrund von Fixkosten bei der Emission entstehen, verantwortlich sein. Zudem wurden die Variablen Financial-Distress Kosten und Agency-Kosten mit in das Regressionsmodell aufgenommen. Diese Determinanten gehen auf die Trade-Off-Theorie zurück, in welcher sie die optimale Kapitalstruktur mitbestimmen. Es kann der Tabelle 5 entnommen werden, dass beide einen signifikanten Wertbeitrag zur abnormalen Rendite liefern. Dies lässt die Vermutung aufkommen, dass die Trade-Off-Theorie trotz anders gerichteter durchschnittlicher Werteffekte Erklärungskraft be- 
sitzt. Die negativen Renditen lassen sich dann damit begründen, dass sich das Unternehmen in Folge der Anleiheplatzierung weiter von der optimalen Kapitalstruktur entfernt.

\begin{tabular}{|c|c|c|c|c|}
\hline \multirow[b]{3}{*}{ Regressionsparameter } & \multicolumn{4}{|c|}{ Ereignisfenster } \\
\hline & \multicolumn{2}{|c|}{$[0]$} & \multicolumn{2}{|c|}{$[-10 ; 0]$} \\
\hline & ßi & $\mathrm{p}$-Wert & ßi & p-Wert \\
\hline Konstante & 0,465 & 0,846 & 6,361 & 0,382 \\
\hline Währung & $-0,087$ & 0,894 & 0,143 & 0,943 \\
\hline Makro-Branche & 0,305 & 0,599 & $-0,063$ & 0,972 \\
\hline Rating & $-0,393$ & 0,620 & $-1,302$ & 0,590 \\
\hline Verwendungszweck & 0,772 & 0,311 & $5,261 * *$ & 0,024 \\
\hline Marktkapitalisierung & $-0,716 * * *$ & 0,001 & $-0,933$ & 0,158 \\
\hline Emissionsvolumen & $0,001 * * *$ & 0,000 & $0,002 * * *$ & 0,002 \\
\hline Freefloat & $0,031 * *$ & 0,002 & $-0,064 * *$ & 0,034 \\
\hline Konjunktur & 8,776 & 0,310 & 2,269 & 0,283 \\
\hline Zinssatz & 0,239 & 0,499 & $-0,121$ & 0,910 \\
\hline $\begin{array}{l}\text { Market to Book } \\
\text { Value }\end{array}$ & $-0,037$ & 0,716 & $-0,476$ & 0,123 \\
\hline Financial Distress & $-0,004$ & 0,586 & $-0,040 *$ & 0,059 \\
\hline Excess-Cash-Flow & 2,431 & 0,337 & $1,809 * *$ & 0,020 \\
\hline R_adj & \multicolumn{2}{|c|}{0,177} & \multicolumn{2}{|c|}{0,085} \\
\hline p Wert des F-Tests & \multicolumn{2}{|c|}{0,000} & \multicolumn{2}{|c|}{0,000} \\
\hline $\begin{array}{l}\text { p Wert des Durbin } \\
\text { Watson Tests }\end{array}$ & \multicolumn{2}{|c|}{0,000} & \multicolumn{2}{|c|}{0,017} \\
\hline
\end{tabular}

$\because *$ signifikant auf dem 1\%-Niveau, ** signifikant auf dem 5\%-Niveau, * signifikant auf dem 10\%-Niveau

Tabelle 5: Ergebnisse der multivariaten Regression

\section{Fazit}

Auch wenn es wegen der grundsätzlich anderen Struktur der Finanzmärkte in Deutschland naheliegend wäre, stellen wir in der vorliegenden Untersuchung keine grossen Unterschiede zu den Ergebnissen anderer Studien aus dem amerikanischen Raum fest. Insgesamt scheint es in Deutschland nicht entscheidend höhere Kosten in Form abnormaler Renditen für die Unternehmen mit sich zu bringen, wenn sie Anleihen begeben, um Fremdkapital aufzunehmen. Die insgesamt negativ ausfallenden Werteffekte für Aktieninhaber können mit Hilfe der Pecking-Order-Theorie oder dem Entfernen von der optimalen Kapitalstruktur im Sinne der Trade-Off-Theorie erklärt werden. Hiernach sieht der Markt die externe Mittelbeschaffung als grundsätzlich schlechter an als mögliche interne Finanzierungsquellen. 
Zudem konnten im Rahmen der detaillierteren Auswertung von Teilstichproben und der multivariaten Regression mehrere Werttreiber identifiziert werden. Für die Erklärung der jeweiligen Einzeleffekte reicht jedoch die reine Fokussierung auf die Pecking-Order-Theorie nicht aus. Hier finden im Einzelfall auch die anderen in der Literatur gängigen Erklärungsansätze ihre Berechtigung. So finden wir auf dem noch jungen Markt für High-YieldAnleihen eine deutlich negativere Rendite als bei Investment-Grade-Titeln. Offenbar wird hier von einem erhöhten Financial Distress Risiko ausgegangen, welches durch die Emission verstärkt wird. Des Weiteren finden wir für Anleihen ausserhalb der Finanzwirtschaft und bei konkreter Angabe eines Verwendungszweckes, der ausdrücklich nicht der reinen Refinanzierung dient, sogar signifikant positive Renditen. Hier kommt der Trade-Offbzw. der Signaling-Theorie eine gewisse Relevanz zu.

Während unsere Studie insgesamt negative Renditen aufzeigt und damit für die PeckingOrder-Theorie spricht, konnten wir Einflussfaktoren ermitteln, die auch positive Renditen für Anleger erwarten lassen. Deshalb lässt sich derzeit nicht abschliessend sagen, welches theoretische Erklärungsmodell schlussendlich auch empirisch das Dominierende ist. Für die Zukunft sehen wir in diesem Bereich Forschungsbedarf, denn insbesondere für kleine Nischenmärkte, wie bspw. dem jungen Markt für Mittelstandsanleihen, sind die Effekte nur unzureichend empirisch belegt, gleichzeitig sind sie aber von hoher Relevanz für die Geschäftsleitung und Anleger. Unsere Ergebnisse legen nahe, dass eine Anleiheemission zur reinen Refinanzierung, bei gleichzeitig hohem Risiko, von Eigenkapitalinvestoren besonders negativ bewertet wird. Auf der anderen Seite können gesunde Unternehmen konkrete Investitionsprojekte fremdfinanzieren und bei Ausnutzung des Tax Shields für Ihre Anleger einen echten Mehrwert generieren.

\section{Literaturhinweise}

Akerlof, G.A. (1970): The Market for "Lemons”: Quality Uncertainty and the Market Mechanism, in: The Quarterly Journal of Economics, Jg. 84, Nr. 3, S. 488-500.

Arrow, K.J. (1972): Gifts and Exchanges, in: Philosophy \& Public Affairs, Jg. 1, Nr. 4, S. 343-362.

Berkman, H./Bradbury, M. (1996): Empirical evidence on the corporate use of derivates, in: Financial Management, Jg. 25, S. 5-13.

Betzer, A./Limbach, P. (2010): Ankündigungseffekte der Emission von High-Yield Bonds in Europa, in: Kredit und Kapital, Jg. 43, Nr. 2, S. 243-270.

Bharath, S./Pasquariello, P./Wu, G. (2009): Does asymmetric information drive capital structure decisions?, in: Review of Financial Studies, Jg. 22, S. 3211-3243.

Boehmer, E./Musumeci, J./Poulsen, A. (1991): Event-study methodology under conditions of eventinduced variance, in: Journal of Financial Economics, Jg. 30, S. 253-272.

Brounen, D./de Jong, A./Koedijk, K. (2006): Capital structure policies in Europe: Survey evidence, in: Journal of Banking \& Finance, Jg. 30, S. 1409-1442.

Choe, H./Masulis, R./Nanda, V. (1993): Common stock offerings across the business cycle, in: Journal of Empirical Finance, Jg. 1, S. 3-31.

Dann, L./Mikkelson, W. (1984): Convertible Debt Issuance, Capital Structure Change and Financing-related Information, in: Journal of Financial Economics, Jg. 13, S. 157-186.

Drobetz, W./Fix, R. (2005): What are the Determinants of the Capital Structure? Evidence from Switzerland, in: Swiss Journal of Economics and Statistics, Jg. 151, S. 71-111. 
Eckbo, B. (1986): Valuation Effects of corporate debt, in: Journal of Financial Economics, Jg. 15, S. 119-151.

Fama, E./Fisher, L./Jensen, M./Roll, R. (1969): The Adjustment of Stock Prices to New Information, in: International Economic Review. Jg. 25, S. 1-27.

Fama, E./French, K. (2002): The Equity Premium, in: The Journal of Finance, Jg. 57, Nr. 2, S. 637-659.

Franke, G./Hax, H. (2004): Finanzwirtschaft des Unternehmens und Kapitalmarkt, Berlin.

Graham, J.R./Harvey, C.R. (2001): The Theory and Practice of Corporate Finance. Evidence from the Field, in: Journal of Financial Economics, Jg. 60, S. 187-243.

Graham, J.R./Rogers, D.A. (2002): Do Firms Hedge in Response to Tax Incentives?, in: Journal of Finance, Jg. 57, S. 815-839.

Harris, M./Raviv, A. (1991): The Theory of Capital Structure, in: The Journal of Finance, Jg. 46, Nr. 1, S. 297-355.

Howton, S./Perfect, S. (1998): The Market Reaction to Straight Debt Issues: The Effects of Free Cash Flow, in: Journal of Financial Research, Jg. 21, S. 219-228.

Huang, R./Ritter, J. (2009): Testing Theories of Capital Structure and Estimating the Speed of Adjustment, in: Journal of Financial and Quantitative Analysis, Jg. 44, S. 237-271.

Kraus, A./Litzenberger, R. (1973): A State Preference Model of Optimal Financial Leverage, in: Journal of Finance, Jg. 28, S. 911-922.

Krishnaswami, S./Yaman, D. (2007): Contracting costs and the window of opportunity for straight debt issues, in: Journal of Banking \& Finance, Jg. 31, S. 869-888.

MacKinlay, A.C. (1997): Event studies in economics and finance, in: Journal of economic literature, Jg. 35, Nr. 1, S. 13-39.

Marsh, P. (1982): The choice between Debt and Equity, in: Journal of Finance, Jg. 37, S. 121-144.

Mikkelson, W./Partch, M. (1986): Valuation Effects of Security Offerings and the Issuance Process, in: Journal of Financial Economics, Jg. 15, S. 31-60.

Miller, M. (1977): Debt and Taxes, in: The Journal of Finance, Jg. 32, Nr. 2, S. 261-275.

Miller, M./Rock, K. (1985): Dividend Policy under Asymmetric Information, in: The Journal of Finance, Jg. 40, Nr. 4, S. 1031-1051.

Modigliani, F./Miller, M. (1958): The Cost of Capital, Corporation Finance and the Theory of Investment, in: The American Economic Review, Jg. 48, Nr. 3, S. 261-297.

Moore, G. (1980): Business Cycles, Inflation, And Forecasting. National Bureau of Economic Research Studies in Business Cylces, Ballinger Publishing Company.

Myers, S. (2001): Capital Structure, in: The Journal of Economic Perspectives, Jg. 15, Nr. 2, S. 81-102.

Myers, S./Majluf, N. (1984): Corporate Financing and Investment Decisions when Firms have Information that Investors Do Not Have, in: Journal of Financial Economics, Jg. 13, Nr. 2, S. 187-221.

Popescu, L./Visinescu, S. (2009): A Review of the Capital Structure Theories, in: Annals of Faculty of Economics, Jg. 3, Nr. 1, S. 315-320.

Psillaki, M./Daskalakis, N. (2009): Are the determinants of capital structure country or firm specific?, in: Small Business Economics, Jg. 33, Nr. 3, S. 319-333.

Rajan, R./Zingales, L. (1995): What Do We Know about Capital Structure? Some Evidence from International Data, in: The Journal of Finance, Jg. 50, Nr. 5, S. 1421-1460. 
Ross, S. (1977): The Determination of Financial Structure: The Incentive-Signalling Approach, in: The Bell Journal of Economics, Jg. 8, Nr. 1, S. 23-40.

Sharpe, W. (1963): A Simplified Model for Portfolio Analysis, in: Management Science, Jg. 9, S. 277-293.

Shyam-Sunder, L. (1991): The Stock Price Effect of Risky versus Safe Debt, in: Journal of Financial and Quantitative Analysis, Jg. 26, Nr. 4, S. 549-558.

Smith, C./Stulz, R. (1985): The determinants of firms' hedging policies, in: Journal of Financial and Quantitative Analysis, Jg. 20, S. 391-405.

Spence, M. (1973): Job Market Signaling, in: Quarterly Journal of Economics, Band 87, Nr. 3, S. 355-374.

Stiglitz, J. (1969): A re-examination of the Modigliani-Miller theorem, in: American Economic Review, Jg. 59, S. 784-793.

Stiglitz, J.E. (1974): On the irrelevance of corporate financial policy, in: American Economic Review, Jg. 64, S. 851-866.

Taggart, R. (1977): A Model of Corporate Financing Decisions, in: Journal of Finance, Jg. 32, S. $1467-1487$.

Warner, J.B. (1977): Bankruptcy Costs: Some Evidence, in: The Journal of Finance, Jg. 32, Nr. 2, S. 337-347.

Christian Babl, Dipl.-Wirtsch.-Ing., ist wissenschaftlicher Mitarbeiter am Lehrstuhl für Unternehmensfinanzierung an der TU Darmstadt.

Andreas Fausel und Leonard Kuhlmann sind Mitarbeiter am Lehrstuhl für Unternehmensfinanzierung an der TU Darmstadt.

Dirk Schiereck, Prof. Dr., ist Lehrstuhlinhaber des Fachgebiets Unternehmensfinanzierung an der TU Darmstadt.

Anschrift: Fachgebiet Unternehmensfinanzierung, Fachbereich Rechts- und Wirtschaftswissenschaften, Technische Universität Darmstadt, Hochschulstr. 1, 64289 Darmstadt; Tel.: +49 (0)6151-16-64531; E-Mail: babl@bwl.tu-darmstadt.de 